\title{
Predictive Validity of a Thigh-Worn Accelerometer METs Algorithm in 5- to 12-Year-old Children
}

\author{
Christiana M.T. van Loo, Anthony D. Okely, Marijka Batterham, Tina Hinkley, Ulf Ekelund, \\ Soren Brage, John J. Reilly, Gregory E. Peoples, Rachel Jones, Xanne Janssen, and Dylan P. Cliff
}

\begin{abstract}
Background: To validate the activPAL3 algorithm for predicting metabolic equivalents (TA $\mathrm{METs}_{\text {) }}$ and classifying MVPA in 5- to 12-year-old children. Methods: Fifty-seven children (9.2 \pm 2.3 y, $49.1 \%$ boys) completed 14 activities including sedentary behaviors (SB), light (LPA) and moderate-to-vigorous physical activities (MVPA). Indirect calorimetry (IC) was used as the criterion measure. Analyses included equivalence testing, Bland-Altman procedures and area under the receiver operating

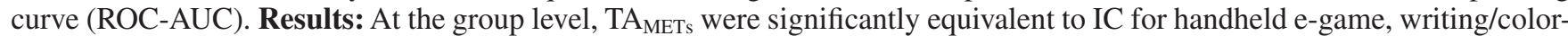
ing, and standing class activity $(P<.05)$. Overall, TA ${ }_{\text {METs }}$ were overestimated for SB $(7.9 \pm 6.7 \%)$ and LPA $(1.9 \pm 20.2 \%)$ and underestimated for MVPA $(27.7 \pm 26.6 \%)$; however, classification accuracy of MVPA was good $($ ROC-AUC $=0.86)$. Limits of agreement were wide for all activities, indicating large individual error (SB: $-27.6 \%$ to $44.7 \%$; LPA: $-47.1 \%$ to 51.0\%; MVPA: $-88.8 \%$ to $33.9 \%$ ). Conclusions: $\mathrm{TA}_{\mathrm{METs}}$ were accurate for some SB and standing, but were overestimated for overall SB and LPA, and underestimated for MVPA. Accuracy for classifying MVPA was, however, acceptable.
\end{abstract}

Keywords: energy expenditure, physical activity, accelerometry, calorimetry, sedentary behavior

Accurate measurement of both sedentary behaviors (SB) and moderate-to-vigorous physical activities (MVPA) is needed to investigate the independent effect of these behaviors on children's health. It is preferable to use 1 monitor to objectively measure both behaviors to minimize participant burden. The activPAL3 (PAL Technology Ltd., Glasgow, Scotland) is a thigh-worn activity monitor that uses triaxial acceleration data $(20 \mathrm{~Hz})$ to assess the position (with respect to gravity) and movement of the limb. Placement on the thigh assists in overcoming difficulties in differentiating between SB and standing or some light-intensity physical activities (LPA), which is common to data analysis approaches used with hip-worn monitors. ${ }^{1}$ The activPAL3 software classifies periods spent sitting/lying, standing or stepping. For studies of physical activity behaviors and obesity prevention in children, it would be useful if activPAL3 data could also accurately assess time spent in MVPA and estimate metabolic equivalents (METs). The activPAL3 provides a MET estimate $\left(\mathrm{TA}_{\mathrm{METs}}\right)$ using a proprietary algorithm, based on default values for each posture combined with step rate and duration of the activity. Previous studies have validated the TA $\mathrm{AETs}_{\mathrm{M}}$ algorithm

van Loo (cmtv1646@uowmail.edu.au), Okely, Jones, and Cliff are with the Early Start Research Institute and the Illawarra Health and Medical Research Institute, Faculty of Social Sciences; Batterham is with the Statistical Consulting Centre; Peoples is with the School of Medicine, Faculty of Science Medicine and Health; University of Wollongong, Australia. Hinkley is with the Institute for Physical Activity and Nutrition (IPAN), School of Exercise and Nutrition Sciences, Deakin University, Australia. Ekelund is with the Dept of Sports Medicine, Norwegian School of Sport Sciences and with the Medical Research Council, Institute of Metabolic Science, Cambridge, United Kingdom. Brage is with the Medical Research Council, Institute of Metabolic Science, Cambridge, United Kingdom. Reilly and Janssen are with the University of Strathclyde, School of Psychological Science and Health, Glasgow, Scotland, UK in 4- to 6-year-olds ${ }^{2}$ and in 15 - to 25 -year-old females. ${ }^{3}$ Thighaccelerometry has shown promising results for assessing SB in 9- to 10-year-olds. ${ }^{4}$ However, to our knowledge, no studies have evaluated $\mathrm{TA}_{\mathrm{METs}}$ algorithm in school-aged children. Therefore, the aim of this study was to examine the predictive validity of $\mathrm{TA}_{\mathrm{METs}}$ algorithm and the accuracy for classifying MVPA in 5- to 12-year-old children.

\section{Methods}

Fifty-seven 5- to 12-year-old children, without physical or health conditions that would affect participation in physical activity, were recruited as part of an activity monitor validation study. The study was approved by the University of Wollongong Health and Medical Human Research Ethics Committee. Parental written consent and participant verbal assent were obtained before participation.

Participants were required to visit the laboratory on 2 occasions. Anthropometric measures were completed using standardized procedures after which BMI $\left(\mathrm{kg} / \mathrm{m}^{2}\right)$ and weight status ${ }^{5}$ were calculated. Children completed a protocol of 14 semistructured 5-min activities including SB, LPA, and MVPA, described elsewhere. ${ }^{6}$ Activities were categorized as SB, LPA and MVPA for descriptive purposes based on the Compendium of Energy Expenditure for Youth. ${ }^{7}$

At each visit, children were fitted with an activPAL3 placed midanteriorly on the right thigh. The activPAL3 is a small and lightweight $(53 \times 35 \times 7 \mathrm{~mm}, 15.0 \mathrm{~g})$ single unit triaxial accelerometer. The activPAL3 software provides an indirect estimate of TA $\mathrm{T}_{\mathrm{METs}}$ based on default values for sitting/lying (1.25 MET), standing (1.40 MET) and stepping at 120 steps per minute (4 MET). Energy expenditure for cadences of greater or less than 120 steps per minute (spm) are calculated using the formula: MET $\cdot \mathrm{h}^{-1}=(1.4 \times \mathrm{d})+(4-1.4) \times(\mathrm{c} \div 120) \times$ $\mathrm{d}$, in which $\mathrm{c}=$ cadence $(\mathrm{spm}), \mathrm{d}=$ activity duration (hours). Software version 7.2.32 was used to export $\mathrm{TA}_{\mathrm{METs}}$ in 15-second epochs.

Oxygen consumption $\left(\mathrm{O}_{2}\right)$ and carbon dioxide production $\left(\mathrm{CO}_{2}\right)$ were assessed using a portable breath-by-breath respiratory 
gas analysis system (MetaMax3B, Cortex, Biophysics, Leipzig, Germany) to provide resting metabolic rate (RMR) and the criterion assessment of physical activity energy expenditure. Before every measurement, the analyzer was calibrated according to the manufacturer's guidelines. At the beginning of each laboratory visit, the thigh-accelerometer and indirect calorimetry (IC) were synchronized with an internal computer clock. RMR was measured at the start of the participant's second visit, while lying down awake on a mattress in supine position with the arms at the sides, resting with minimal movement for $10 \mathrm{~min}$ in a darkened room. Breath-bybreath samples from the data collected between minutes 7.0 and 9.0 were averaged to calculate mean volume of $\mathrm{O}_{2}$. The participants' measured RMR was used to define 1 MET. Metabolic data from the activities were converted into youth METs (scaled to the children's RMR) and averaged over 15-second epochs to align with the thighaccelerometry data using customized software.

Normality of the data were confirmed before analyses. The predictive validity of $\mathrm{TA}_{\mathrm{METs}}$ was examined at the group level using the $95 \%$ equivalence test. To reject the null-hypothesis of the equivalence test, the $90 \%$ confidence interval (CI) of TA $\mathrm{METs}_{\mathrm{M}}$ should entirely fall within the predefined equivalence region of $\pm 10 \%$ of the criterion METs assessed by IC. ${ }^{8}$ Measurement agreement and systematic bias for $\mathrm{TA}_{\mathrm{METs}}$ were evaluated at the individual level using Bland-Altman procedures. Sensitivity, specificity, and area under the receiver operating curve (ROC-AUC) were calculated to evaluate the accuracy for classifying MVPA. A dichotomous coding system was created using 1 for $\geq 3$ METs and 0 for $<3$ METs. ROCAUC values were defined as excellent $(\geq 0.90)$, good (0.80-0.89), fair $(0.70-0.79)$, or poor $(<0.70) .{ }^{9}$ Data reduction and statistical analyses were performed using the statistical computing language R (v.3.1.2) and SPSS (v.19.0).

\section{Results}

Descriptive characteristics of participants are presented in Table 1. All participants completed the protocol. Data from 1 child were entirely excluded from the analyses and data from 4 participants for a total of 9 activities were excluded because of IC failure. Some 15 -second epochs were partly excluded due to misalignment of thigh-accelerometry data with IC data. A total of 16,337 epochs were included for analysis, accounting for $98.8 \%$ of the total data. Mean measured METs for SB, LPA and MVPA activities were 1.17 $\pm 0.08,2.50 \pm 0.78$ and $5.08 \pm 1.15$, respectively. TA $\mathrm{TETs}_{\mathrm{MET}}$ were $1.25 \pm$ $0.0,2.58 \pm 0.94$ and $3.80 \pm 0.23$, respectively. Energy expenditure data per activity are presented in Table 2 for the complete sample, as well as additional data per age group. Statistical analyses were performed for the complete sample (5- to 12-year-olds) only (Table 3). At the group level, $\mathrm{TA}_{\mathrm{METs}}$ were significantly equivalent to IC for handheld e-game $(P=.01)$, writing/coloring $(P<.01)$ and standing $(P=$
$.01)$. All other activities were not equivalent to $\operatorname{IC}(P>.05)$. Mean $\mathrm{TA}_{\text {METs }}$ were underestimated by $7.1 \% \pm 25.9 \%$. TA $\mathrm{METs}_{\mathrm{M}}$ for $\mathrm{SB}$ were slightly overestimated by the algorithm $(7.9 \pm 6.7 \%)$. $\mathrm{TA}_{\mathrm{METs}}$ for slow walk were overestimated by $32.0 \%$; however, $\mathrm{TA}_{\mathrm{METs}}$ for all other LPAs were underestimated by $4.2 \%$ to $10.9 \%$, resulting in a small overestimation of mean TA $\mathrm{TETs}_{\mathrm{MET}}(1.9 \pm 20.2 \%)$ for LPA. TA $\mathrm{METs}_{\mathrm{s}}$ for brisk walk were also overestimated $(21.2 \%)$, whereas $\mathrm{TA}_{\mathrm{METs}}$ for the remaining MVPA activities were underestimated by $34.4 \%$ to $47.3 \%$. On average, TA $_{\text {METs }}$ for MVPA were underestimated by $27.7 \pm 26.6 \%$. Limits of agreement were wide for all activities indicating large individual error. Systematic bias was found for all activities $(P<.001)$, with larger overestimation for low intensities and larger underestimation for high intensities (plots not presented). However, TA $\mathrm{METs}_{\mathrm{M}}$ exhibited good classification accuracy for MVPA $($ ROC-AUC $=0.85$, sensitivity $=0.84$, specificity $=0.87)$.

\section{Discussion}

This study demonstrated that $\mathrm{TA}_{\mathrm{METs}}$ were significantly equivalent to IC for handheld e-game, writing/coloring and standing at the group level, whereas no other activities were equivalent to IC. Overall, $\mathrm{TA}_{\mathrm{METs}}$ for SB were slightly overestimated compared with measured METs. TA $A_{\text {METs }}$ for slow and brisk walking were also overestimated with a larger error. $\mathrm{TA}_{\mathrm{METs}}$ for the remaining LPAs were slightly overestimated compared with measured METs, whereas TA for the remaining MVPA activities were underestimated by a larger amount. Considerable error was demonstrated at the individual level for all activities. Although TA $\mathrm{METs}_{\mathrm{S}}$ for MVPA were underestimated, classification accuracy was acceptable.

Our findings were consistent with previous studies in preschool children $^{2}$ and 15- to 25-year-old females. ${ }^{3}$ These studies reported an overall underestimation of $15 \%$ and $11 \%$ for $\mathrm{TA}_{\mathrm{METs}}$ using thigh-accelerometry, respectively. Although the results in our study demonstrated an overall underestimation of $\mathrm{TA}_{\mathrm{METs}}$, the mean bias was slightly smaller $(7.1 \% \pm 25.9 \%)$ than previous studies. Janssen et $\mathrm{al}^{2}$ reported an overestimation of $6 \%$ for SB and an underestimation of $15.3 \%$ and $32.8 \%$ for LPA and MVPA, respectively, among 4 to 6 year-old children. These values are similar to an overestimation of $7.9 \%$ for SB in our study and underestimation of $27.7 \%$ for MVPA. In contrast with Janssen et $a{ }^{2}{ }^{2}$ we found an overestimation of $1.9 \%$ for LPA. However, when excluding slow walk, the TA for remaining LPAs were underestimated by $4.2 \%$ to $10.9 \%$. Harrington et $\mathrm{al}^{3}$ demonstrated that $\mathrm{TA}_{\mathrm{METs}}$ during walking at lower speed was overestimated, whereas $\mathrm{TA}_{\mathrm{METs}}$ during higher walking speeds were underestimated. This is in line with the overestimation at the lower intensities and underestimation during higher intensities found in our study and by Janssen et al. ${ }^{2}$ The overestimated TA $\mathrm{METs}_{\mathrm{M}}$ during over-ground brisk walk in our study seems to contradict the findings from Harrington et $\mathrm{al}^{3}$ at higher treadmill walking speeds,

Table 1 Descriptive Characteristics of the Participants

\begin{tabular}{lcccccc}
\hline & $\mathbf{n}$ & Age $(\mathbf{y})$ & Height $(\mathbf{c m})$ & Weight $(\mathbf{k g})$ & BMI $\left(\mathbf{k g} \cdot \mathbf{m}^{-2}\right)$ & BMI $(\mathbf{p e r c e n t i l e )}$ \\
\hline Total & 57 & $9.2 \pm 2.3$ & $135.9 \pm 14.6$ & $32.7 \pm 10.9$ & $17.1 \pm 2.5$ & $53.2 \pm 28.6$ \\
Girls & 29 & $8.9 \pm 2.1$ & $134.4 \pm 14.4$ & $30.6 \pm 9.5$ & $16.4 \pm 2.1$ & $46.7 \pm 26.6$ \\
Boys & 28 & $9.5 \pm 2.4$ & $137.5 \pm 14.6$ & $34.8 \pm 12.0$ & $17.8 \pm 2.8$ & $59.9 \pm 29.4$ \\
5- to 9-year-olds & $32(20 \mathrm{~F}, 12 \mathrm{M})$ & $7.5 \pm 1.5$ & $126.4 \pm 10.5$ & $26.4 \pm 6.7$ & $16.2 \pm 1.9$ & $54.3 \pm 27.3$ \\
10- to 12-year-olds & $25(9 \mathrm{~F}, 16 \mathrm{M})$ & $11.3 \pm 1.0$ & $148.2 \pm 8.8$ & $40.7 \pm 10.0$ & $18.2 \pm 2.9$ & $51.7 \pm 30.6$ \\
\hline
\end{tabular}

Abbreviations: BMI, body mass index; F, female; M, male. 


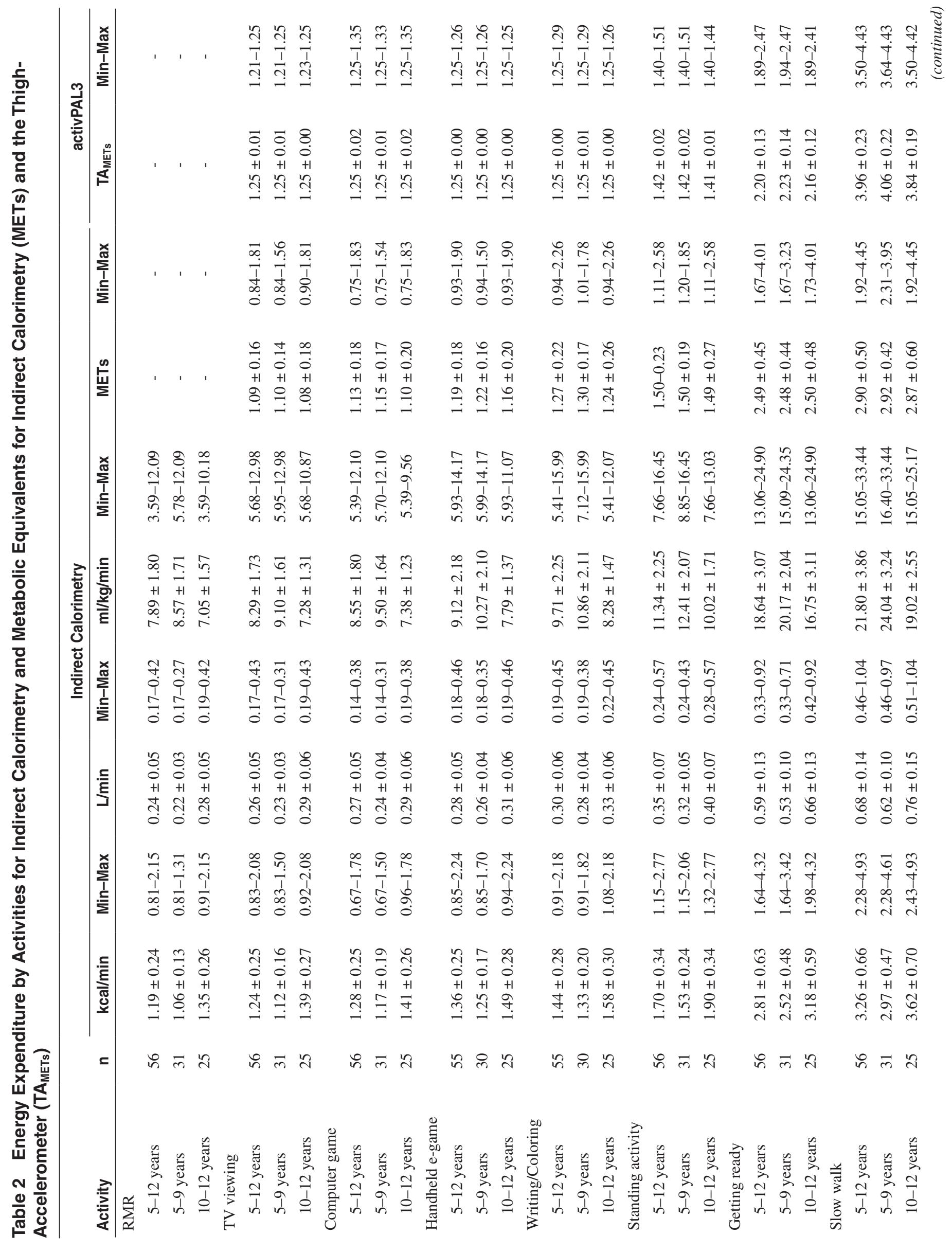




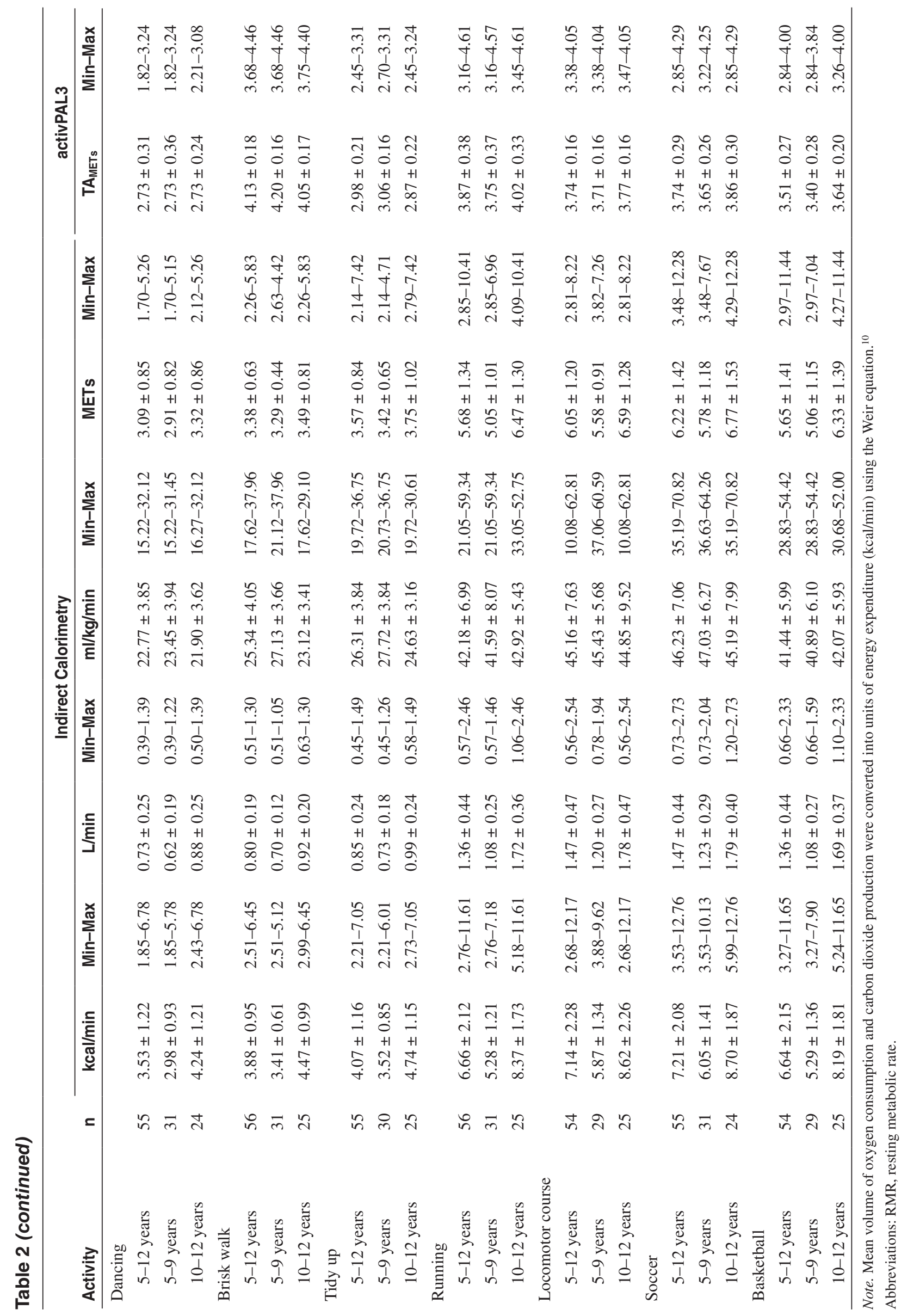


Table 3 Statistical Analyses for the Measurement Agreement of Metabolic Equivalents for Indirect Calorimetry and the Thigh-Accelerometer (TA $\left.\mathrm{METS}_{\mathrm{S}}\right)$

\begin{tabular}{|c|c|c|c|c|c|c|c|}
\hline \multirow[b]{2}{*}{ Activity } & \multirow[b]{2}{*}{$\mathbf{n}$} & \multicolumn{3}{|c|}{ Equivalence testing ${ }^{\mathrm{a}}$} & \multicolumn{3}{|c|}{ Bland-Altman analysis ${ }^{b}$} \\
\hline & & $90 \% \mathrm{ClTA}_{\mathrm{METS}}$ & Equivalence zone IC & $\boldsymbol{P}$ & Mean bias (\%) & $95 \%$ LoA & Slope $\boldsymbol{P}$ \\
\hline TV & 56 & $1.25-1.25$ & $0.98-1.20$ & 0.992 & -14.50 & -39.13 to 10.12 & 0.0001 \\
\hline Computer game & 56 & $1.25-1.26$ & $1.02-1.24$ & 0.715 & -11.80 & -42.58 to 18.99 & 0.0001 \\
\hline Handheld e-game & 55 & $1.25-1.25$ & $1.07-1.31$ & 0.011 & -6.00 & -34.00 to 22.06 & 0.0001 \\
\hline Writing/coloring & 55 & $1.25-1.25$ & $1.14-1.40$ & 0.000 & 0.61 & -29.64 to 30.86 & 0.0001 \\
\hline Standing activity & 56 & $1.41-1.42$ & $1.35-1.65$ & 0.011 & 4.21 & -23.21 to 31.63 & 0.0001 \\
\hline Getting ready & 56 & $2.17-2.23$ & $2.24-2.74$ & 0.752 & 10.86 & -25.34 to 47.06 & 0.0001 \\
\hline Slow walk & 56 & $3.91-4.01$ & $2.61-3.19$ & 1.000 & -32.02 & -64.93 to 0.89 & 0.0001 \\
\hline Dancing & 55 & $2.66-2.80$ & $2.78-3.40$ & 0.684 & 9.24 & -33.03 to 51.50 & 0.0001 \\
\hline Brisk walk & 56 & $4.09-4.17$ & $3.04-3.72$ & 1.000 & -21.24 & 17.36 to 12.77 & 0.0001 \\
\hline Tidy up & 55 & $2.93-3.02$ & $3.21-3.93$ & 0.979 & 15.82 & -25.61 to 57.24 & 0.0001 \\
\hline Basketball & 54 & $3.45-3.57$ & $5.00-6.12$ & 1.000 & 43.88 & 18.36 to 79.87 & 0.0001 \\
\hline Running & 56 & $3.79-3.96$ & $5.11-6.25$ & 1.000 & 35.35 & -6.41 to 77.11 & 0.0001 \\
\hline Locomotor course & 54 & $3.70-3.77$ & $5.11-6.25$ & 1.000 & 44.92 & 5.00 to 84.83 & 0.0001 \\
\hline Soccer & 55 & $3.67-3.80$ & $5.60-6.84$ & 1.000 & 47.26 & 9.29 to 85.22 & 0.0001 \\
\hline
\end{tabular}

Abbreviations: LoA, limits of agreement; CI, confidence interval; IC, indirect calorimetry.

a 95\% equivalence test for $\mathrm{TA}_{\mathrm{METs}}$. Methods are equivalent if $90 \%$ confidence intervals lie entirely within the equivalence region of indirect calorimetry.

${ }^{\mathrm{b}}$ Mean bias was calculated as: measured METs $-\mathrm{TA}_{\mathrm{METs}}$; a positive value indicates underestimation of $\mathrm{TA}_{\mathrm{MET} s}$; a negative value indicates overestimation $\mathrm{TA} \mathrm{AETS}_{\mathrm{M}}$.

which might be explained by differences in the age of the samples and protocols. Despite the underestimation of TAMETs for MVPA activities, the algorithm showed good classification accuracy for this intensity when using a 3-MET threshold. This was likely because the 15-second MET values were consistently underestimated, but were typically above 3 METs and so accurately categorized as MVPA. Therefore, the monitor might be appropriate to use for classification of MVPA in combination with estimating SB in school-aged children.

As suggested in previous studies, ${ }^{2,3}$ the predictive validity of the proprietary algorithm might be affected because step rate is included as the only independent variable. A study by Aminian et $\mathrm{al}^{4}$ validated the step count function of the monitor in 9- to 10-yearold children. Step counts were overestimated in over-ground fast walking, which might explain the overestimated $\mathrm{TA}_{\mathrm{METs}}$ during this activity in our protocol. Other potential predictors such as thighaccelerometry counts, ${ }^{3}$ in addition to age, height, and weight might improve accuracy.

A strength of this study is the large sample size including a broad age range and a wide range of semistructured lifestyle activities. A potential limitation was that RMR values were measured preexercise and might not reflect true rest. Furthermore, findings in this study need to be confirmed during less structured activities or under free-living conditions.

\section{Conclusion}

This study in school-aged children suggests that the $\mathrm{TA}_{\mathrm{MET}}$ algorithm performed reasonably well at the group level for some SB activities and standing, but estimates were inaccurate for higher intensities and large variability was found at the individual level. Therefore, the algorithm may need further development and improvement before it can be used to accurately estimate METs. Although estimates of METs were inaccurate for MVPA, classification accuracy for MVPA was good when using a 3 METs threshold. This suggests that the $\mathrm{TA}_{\mathrm{METs}}$ algorithm may be suitable for classifying MVPA in school-aged children.

\section{Acknowledgments}

We would like to thank participating children and parents, and Melinda Smith for her assistance with recruitment and data collection. This study was funded by the National Heart Foundation of Australia (G11S5975). DPC was funded by a National Heart Foundation of Australia Postdoctoral Research Fellowship (PH 11S 6025) and an Australian Research Council (ARC) Discovery Early Career Researcher Award (DE140101588). TH is funded by a National Health and Medical Research Council (NHMRC) Early Career Fellowship (APP1070571). ADO is supported by a National Heart Foundation of Australia Career Development Fellowship (CR11S 6099).

\section{References}

1. anssen X, Cliff DP. Issues related to measuring and interpreting objectively measured sedentary behavior data. Meas Phys Educ Exerc Sci. 2015;19(3):116-124. doi:10.1080/1091367X.2015.1045908

2. Janssen X, Cliff DP, Reilly JJ, et al. Validation and calibration of the activPAL for estimating METs and physical activity in 4-6 year olds. J Sci Med Sport. 2014;17(6):602-606. PubMed doi:10.1016/j. jsams.2013.10.252

3. Harrington DM, Welk GJ, Donnelly AE. Validation of MET estimates and step measurement using the ActivPAL physical activity logger. $J$ Sports Sci Mar. 2011;29(6):627-633. PubMed

4. Aminian S, Hinckson EA. Examining the validity of the ActivPAL monitor in measuring posture and ambulatory movement in children. Int J Behav Nutr Phys Act. 2012;9:119. PubMed doi:10.1186/14795868-9-119 
5. Kuczmarski RJ, Ogden CL, Guo SS, et al. 2000 CDC Growth Charts for the United States: methods and development. Vital Health Stat 11. 2002;246:1-190. PubMed

6. van Loo CMT, Okely AD, Batterham M, et al. Validation of the SenseWear Mini activity monitor in 5-12-year-old children. J Sci Med Sport. 2016;pii:S1440-2440(16)30057-3. PubMed

7. Ridley K, Ainsworth BE, Olds TS. Development of a compendium of energy expenditures for youth. Int J Behav Nutr Phys Act. 2008;5(1):45-53. PubMed doi:10.1186/1479-5868-5-45
8. Batterham MJ, Van Loo C, Charlton KE, Cliff DP, Okely AD. Improved interpretation of studies comparing methods of dietary assessment: combining equivalence testing with the limits of agreement. Br J Nutr. 2016;115(07):1273-1280. PubMed doi:10.1017/S0007114516000040

9. Metz CE. Basic principles of ROC analysis. Semin Nucl Med. 1978;8(4):283-298. PubMed doi:10.1016/S0001-2998(78)80014-2

10. Weir JBV. New methods for calculating metabolic rate with special reference to protein metabolism. J Physiol. 1949;109(1-2):1. PubMed doi:10.1113/jphysiol.1949.sp004363 